\title{
Mechanistic Explanation: Some Limits and their Significance
}

\author{
Dominie Dessaix
}

\section{Abstract}

In this essay I discuss mechanistic explanation (ME), which is a type of explanation characteristic of, though not exclusive to, the biological sciences. ME differs radically from the deductive-nomological (DN) model of explanation around which, until recently, the received view of explanation in the philosophy of science was centered. The aim of the essay is firstly to present and explain $\mathrm{ME}$, and compare it to the traditional DN account. I also consider three potential limits of ME that have been discussed in the literature, relating to ME's scope, generality, and the completeness of the models it generates. While all of these point to real features of ME, I argue that they are not problematic because of the nature of the phenomena ME aims to explain, as well as the nature of scientific explanation more generally. My conclusion is that no limit to $\mathrm{ME}$ is as serious as the consequences of not giving this important kind of scientific explanation a central place in our account of how science explains the world.

Explanation being one of the central aims of science is perhaps obvious. It subsumes some of the other aims that are attributed to science (e.g., understanding how the world works, uncovering reality), and is a favourable companion to others (e.g. successful prediction ${ }^{1}$ ). Far less obvious is what constitutes a good scientific explanation, and whether one account could fit all sciences, or even all the kinds of phenomena in a single science. Mechanistic explanation (ME) is a type of explanation prevalent in, though not exclusive to, the life sciences. In what follows, I outline a general account of $\mathrm{ME}^{2}$ provide some examples, and briefly contrast it with the deductive-nomological ${ }^{3}(\mathrm{DN})$ account of explanation: what was, until recently, 'the received view of scientific explanation in philosophy' (Bechtel \& Abrahamsen 2005: 421), despite some serious criticism (e.g., Cummins

\footnotetext{
1 Of course, prediction does not have to involve explanation: one might discover some pattern in a phenomenon and make successful predictions without correctly explaining it.

2 It is a version based on Craver's $(2002,2007)$ causal-mechanistic explanation. Though there are other accounts of ME (e.g., see Glennan 1996, 2002), the differences are not directly relevant to the purpose of this paper.

3 Deductive means 'relating to logical deduction'; nomological means 'relating to laws'.
} 
2000; Kim 1962). I then consider three potential limits to ME. Firstly, I argue that the probable restriction of ME's scope to certain areas of science, or to some kinds of phenomena in those areas, doesn't threaten the validity of ME where it is found fruitful. Secondly, I look at the objection that MEs, unlike explanations that appeal to scientific laws (i.e. DN), cannot properly generalise over the phenomena they describe. I argue that this is also unproblematic, and may even be construed as a virtue of ME. Thirdly, I look at Nicholson's (2012) argument, which states that if we think of biological mechanisms as real world entities, the mechanistic explanations that describe them will fail to be complete, because they must always abstract away from the mechanism's 'organismic context'. I show firstly that Nicholson's discussion conflates real world mechanisms with the models that describe them, and then argue that lack of completeness is not a serious objection to $\mathrm{ME}$, or indeed any scientific explanation. I conclude that although we should be mindful of ME's limited scope, generality and completeness, these features do not pose problems for the use of ME for at least certain phenomena in the life sciences, and perhaps beyond.

It is first necessary to characterise ME. Minimally, a mechanistic explanation involves presenting a model of the mechanism (the explanans ${ }^{4}$ ) responsible for some phenomenon of interest (the explanandum ${ }^{5}$ ). A mechanism is an entity or structure, such as an organ (though a mechanism need not be delineated by such clear physical boundaries ${ }^{6}$ ) that performs a function by virtue of its component parts, operations and, importantly, their spatial and temporal organisation (Bechtel \& Abrahamsen 2005: 421-23). That is, a mechanism does not just consist in its physical parts, but in the 'organised interplay' of the activities of those parts (Soom 2012: 656). Examples of phenomena (i.e., functions or behaviours performed by mechanisms) include such things as the heart's pumping blood, metabolism and memory. Explanation is achieved by a mechanistic model 'rendering a phenomenon intelligible' by showing how the organised interaction of the component parts produces the phenomenon of interest (McManus 2012: 533), thereby showing why the phenomenon happens in the way that it does.

Consider, for example, the function of the heart to pump blood. What constitutes a mechanistic explanation of the heart qua blood-pumper are the parts of heart (atria, ventricles, valves, etc.), the operations performed by these parts (e.g., contraction and relaxation by the four chambers), and their organisation (blood flows from each atrium to valve and ultimately into the circulatory system) (Bechtel \& Abrahamsen 2005: 424). Spatial memory is also, in principle,

4 Lat. that which does the explaining.

5 Lat. that which is to be explained; i.e., the thing or event that requires explanation.

6 Craver (2007: 141-44) discusses how delineating the boundaries of a given mechanism must be done with respect to explanatory relevance, not just on the basis of compartmental (i.e., physical) boundaries. 
amenable to ME (though it doesn't yet have a completed model). Knowing that the hippocampus is crucially involved in our capacity to represent the spatial layout of our environment means we know that the hippocampus is a crucial component of the mechanism responsible for behaviours related to the processing and memorisation of spatial features of the environment (Soom 2012: 656; Craver 2002: 89).

Now, before considering the possible limits of ME, I shall describe the DN account of explanation, and draw some comparisons between it and $\mathrm{ME}$, in order to demonstrate how the latter is distinctive. Just as in ME, a DN explanation consists in an explanandum (on the DN view, a sentence describing the phenomenon to be explained) and an explanans (the sentences that do the explaining). DN requires the fulfillment of four conditions: the explanans must 1) contain a statement of a law of nature, 2) include an empirical statement amenable to testing and, 3) consist only in true statements; and, the explanandum must 4) follow logically from the explanans (Fetzer 2013). The central thesis is that a phenomenon is explained by subsumption under natural law (Hempel 1966: 50ff). For example, one could derive the position of Mars at some future time (the explanandum) with an argument (the explanans) containing Newton's laws of motion, the inverse square law and empirical statements about the initial conditions, such as the mass, position and velocity of both Mars and the sun (Woodward 2011). Contrast this with Bechtel \& Abrahamsen's (2005: 422) example of metabolism (cellular respiration), which if made to fit into the DN model of explanation, would use law-like statements like the following:

Under specified conditions in the last phase of cellular respiration, the ratio of oxygen molecules consumed to ATP molecules produced does not exceed 1:3. (Bechtel \& Abrahamsen 2005: 422)

Though true, such a statement, and the DN explanation it would form part of, can do little explanatory work. By not detailing the mechanism of metabolism in which oxygen and ATP molecules play a part, such an explanation cannot tell us what lies behind that ratio. ${ }^{7}$ For these cases, what we want to show is not 'that a phenomenon fits within the nomic ${ }^{8}$ nexus' but rather 'how a phenomenon fits into the causal nexus' (Craver 2007: 39 on Salmon 1984). That is, what is interesting and explanatory here is finding out what causes and follows each stage of metabolism, and how all the components and processes relate to one another. We want to know, in short, how cellular respiration works. Law-like generalisations such as those above are not illuminating. Whatever the virtues

7 See Bechtel \& Abrahamsen (2005) for more information on the ME of cellular respiration.

8 Here 'nomic' just means 'relating to a law'. 
of the DN model in some areas of science (or otherwise, for the DN explanation has its own issues ${ }^{9}$ ), for these sorts of biological processes it doesn't yield satisfactory explanations.

There are three further features of ME that make it appropriate where it is most notably employed, viz. the life sciences. Firstly, it is inherently multi-level, aiming to explain higher level phenomena by reference to mechanisms at lower levels (Soom 2012: 657). This means that ME has a recursive structure; i.e., a mechanism can be contained in another mechanism, which can be contained in another mechanism, and so on. The heart, for example, is both a mechanism for pumping blood and a component of the larger mechanism of the circulatory system (Bechtel \& Abrahamsen 2005: 425). DN explanations, as separate arguments, do not give this sort of unity of explanation, which is so appropriate for biological phenomena. Secondly, ME is flexible with regard to how it is presented: like DN explanations, MEs can be presented linguistically, but they might also employ diagrams, three-dimensional models, computer simulations, and so on. Thirdly, ME is not simply either correct or incorrect, unlike (the deductive part of) a DN explanation must be, and is thus more obviously consistent with actual scientific practice, in the life sciences at least, where mechanistic models are continually filled in and refined (Craver 2007: 113f).

A perspicuous potential limit to ME is its scope: are MEs only applicable to some phenomena in biology? After all, the example of DN discussed above (that of predicting Mars' position) does look like a clear case of subsumption under law, and not at all like ME terrain. Furthermore, current accounts of ME may not even work for all of biology. For example, McManus (2012) argues that causalmechanistic explanations are inconsistent with developmental mechanisms. Yet some restriction of ME's scope to certain fields or phenomenon type is neither worrisome nor surprising. For ME to count as a valid kind of scientific explanation, it is sufficient that it proves fruitful for some phenomena in some sciences. And it is worth bearing in mind that the scope of ME is still an open question: it could turn out that the usefulness of ME extends out from the biological sciences. Ramsey (2008), for example, discusses how organic chemists consider mechanisms explanatory. In any case, the fact that mechanisms are widely and successfully used in explanations in many areas of biology (Brechtel \& Abrahamsen 2005: 421f), coupled with the fact that not all phenomena are amenable to ME (e.g., Glennan's (2002) 'genuinely singular events' ${ }^{\prime 10}$ ), suggests

9 Such as the problem of explanatory irrelevancies: it is possible to create valid DN arguments that patently miss their explanatory target, as in the famous example that, from the law 'All males who take birth control pills regularly fail to get pregnant', and fact 'John Jones is a male who has been taking birth control pills regularly', we can derive the conclusion 'John Jones fails to get pregnant', for which the premises don't offer a satisfactory explanation. See Woodward 2011 for this and other issues faced by the DN model.

10 Glennan (2002: 349f) gives the example of a person first meeting her partner: such events typically 'involve a confluence of events that were not to be expected and will not be repeated', and there isn't a sufficiently 
not that $\mathrm{ME}$ is on the wrong track, but that we require a pluralistic account of explanation. This probable limit of scope accepted, I will move on to consider two possible limits to ME that I think invite more discussion. First, I look at the apparent limited generality offered by ME explanations, especially when contrasted with DN explanations, then move on to consider Nicholson's (2012) argument that an 'ontic' view of biological mechanisms (roughly, conceiving of mechanisms as real things in nature) doesn't sit well with the fact that MEs are always incomplete due to the necessity of abstracting away from the context of the whole organism.

Scientific laws of nature are universally quantified generalisations. Thus explanations appealing to them allow us to generalise to those unobserved cases that the law subsumes. It looks to be a virtue of $\mathrm{DN}$ explanations that generalisation is automatic. Mechanistic models, on the other hand, can be highly 'specific' and 'context-bound' (Bechtel \& Abrahamsen 2005: 437). Scientists working on a given ME might take into account a number of local factors that are only applicable to the single individual or type of entity under study. So, one might ask, how can a mechanistic explanation help us account for what we should expect to find in unobserved cases? Bechtel \& Abrahamsen (2005: 437) suggest that we appeal to an alternative kind of generalisation inspired by prototype and exemplar theories. The general idea of such theories is that an item's membership of a certain category can be a matter of degree, depending on how typical the item is of that category. For example, psychological research conducted by Rosch $(1975,1978)$ showed that we do not treat all bird species as equally good members of the bird category, but instead tend to classify, for example, robins as highly typical members (one of the 'birdiest' bird species), and penguins as highly atypical (considerably less birdy). In a parallel way, we could classify mechanisms as belonging to a more general mechanism-type based on those mechanisms' similarity to one another, and their resemblance to a prototype. This could apply, for example, to the various mechanisms of protein synthesis in different individual organisms and species, and in different cell types of the same species (Bechtel \& Abrahamsen 2005: 438).

So, by allowing ourselves a fuzzier conception of membership based on similarity and prototypicality, we may get sufficient generality of mechanism types, without precluding cases with important variations, whether at the level of the individual, species, or cell type. In addition, I suggest that by embracing a kind of functionalism that brings disparate mechanisms under the same mechanism type as long as they perform an analogous function, we might have another generalising tool, of which the appropriateness in the biological 
sciences has independent empirical support. Consider, for example, that leftand right-handed subjects show different patterns of cerebral lateralisation (i.e., use different neural mechanisms) when performing the same behaviour (i.e., to produce the same phenomenon) (Stoyanov et al. 2012: 1; Sun \& Walsh 2006: 655). This non-automatic, fuzzier sort of generalisation, which might also appeal to functional roles, should be thought of as a consequence of the appropriateness of ME to the scientific domain, where it is most often used. After all, the biological world is teeming with variation, so we shouldn't expect to find a single mechanistic explanation to work for countless slightly varied mechanisms of, say, protein synthesis. The limited generality of ME is thus more a virtue of rather than a limit to ME's explanatory power.

Now I shall consider a final possible limit to ME, that of completeness, as discussed by Nicholson (2012). So far I have been talking about mechanisms both as real things in nature, and as described by models in scientific explanation. Nicholson (2012), however, has argued against what he calls an 'ontic conception of mechanisms' (conceiving of the mechanisms described by ME as real entities in the world), at least in the context of biology. He points out that biological mechanisms are never described completely by MEs, which abstract away from the 'organismic context' necessary for the proper function of the mechanism (2012: 159). That is, in actual scientific practice, biologists only include in a given ME those features they deem most relevant to bringing about the phenomenon of interest, necessarily presupposing many features of the wider context that make the mechanism work as it does. Referring to Craver's own normative requirement on ME: that it must fully account for the explanandum behaviour, not merely a subset of its features (Craver 2007: 161), Nicholson then argues that on these terms, MEs are necessarily incomplete (2012: 159).

This leads Nicholson to argue for an 'epistemic' view of mechanisms: we should consider the mechanisms described by ME as 'heuristic models that facilitate the explanation of phenomena' (2012: 158). For if we think of the mechanisms described by our MEs as just approximating what happens in the natural world, then we should expect that those descriptions will be incomplete. While I ultimately agree with taking a roughly 'epistemic' view of ME, Nicholson's discussion unfortunately conflates the real world mechanisms that ME tries to explain, with the mechanistic models that figure in our explanations. He defines a biological 'causal mechanism' as 'a step-by-step explanation of the mode of operation of a causal process that gives rise to a phenomenon of interest' (2012: 153). The latter is clearly a definition of a mechanistic explanation, not of a mechanism itself. Nicholson also claims that it is problematic to think of mechanisms as 'real systems in nature' or as 'autonomous complex systems [...] which constitute and operate within the organism' (2012: 159). Yet I take it that mechanisms themselves can and should be seen as real things out there in 
the world, which a mechanistic explanation tries to capture and describe. As for any kind of scientific explanation, ME aims to describe the components, operations and organisation of real entities in nature, entities whose existence is not dependent on the biologists who study them. Though Nicholson nowhere denies this realist position, his talk of 'mechanisms' to mean mechanistic models or explanations runs together issues of scientific realism with the issue with which he is really concerned, namely, the issue of how completely ME capture those real world mechanisms. ${ }^{11}$

But as far as the incompleteness of MEs is concerned, Nicholson's (2012) argument is convincing: we should bear in mind that mechanistic models are just models, and MEs just abstractions of the phenomena they describe. I stress, however, that such incompleteness is not problematic. That we are presently unable to access and specify all the ways in which the organismic context influences individual mechanisms in minuscule detail, and that it may never be possible to do so, says only that we cannot construct 'technically complete' mechanistic models. We can still aim for (and hopefully achieve) high quality sketches, which may even reach a sort of 'practical' rather than 'technical' completeness, in that they may explain the mechanism fully when certain (very many) background conditions are specified (e.g., for the mechanistic explanations of memory, 'the brain must be contained in a living individual of species $x^{\prime}$ ). But more important, I think, is the fact that incompleteness is not an impediment to, but a necessary feature of, many kinds of scientific explanation. If we want to give a satisfactory explanation of a wide range of similar, non-identical instances, our explanation must be sufficiently general, and this simply means abstracting away from the details. We could not, for example, give a mechanistic explanation of heart function in vertebrates (diverse creatures with diverse organismic contexts) without such abstraction. A certain 'incompleteness', then, is necessary for some scientific explanations to be explanatory, and thus does not amount to a serious objection to ME.

This essay has discussed ME, comparing it to the DN model and describing some of the features that make ME suited to the phenomena it has been used to explain, most notably in biology. I considered potential limits, none of which were found to threaten ME's validity as a fruitful sort of scientific explanation. If $\mathrm{ME}$ is indeed limited to some areas of science or some kinds of phenomena, this points to the need for a pluralistic account of explanation, not to some defect of ME. Also, ME's inability to automatically generalise, as the appeal to laws allows

11 There is a genuine debate in the literature between the 'ontic' and 'epistemic' conceptions on ME, but it is rather more subtle than Nicholson's (2012) treatment. Basically, the ontic view says that the mechanism itself being causally responsible for a phenomenon is what explains that phenomenon, whereas the epistemic view of what makes ME explanatory emphasises the role of conveying understanding about how the mechanism produces a phenomenon. See Illari (2013) for discussion and a persuasive argument that these views are not mutually exclusive. 
the DN model to do, does not make ME explanatorily weaker, but different in the kind of generalisation it can provide, which is argued in turn appropriate to the biological phenomena it describes. Finally, I argued that although Nicholson's (2012) presentation of his argument confounds real mechanisms and mechanistic models, I agree with the 'epistemic' view of ME that he suggests, which accepts that ME are necessarily incomplete. I argued, however, that such incompleteness is not problematic, and is indeed an important feature of much scientific explanation. Thus the limit to our understanding of science, if we don't come to grips with this crucial kind of scientific explanation, is far graver than any limit to ME itself.

\section{Bibliography}

Bechtel, W \& Abrahamsen, A (2002), 'Explanation: A Mechanistic Alternative', Studies in History and Philosophy of Science Part C: Studies in History and Philosophy of Biological and Biomedical Sciences, vol. 36, no. 3, pp. 421-41.

Craver, CF (2002), 'Interlevel Experiments and Multilevel Mechanisms in the Neuroscience of Memory', Philosophy of Science, vol. 69, no. S3, pp. S83-S97.

- (2007), Explaining the Brain, Oxford University Press.

Cummins, R (2000), “"How Does it Work" Vs. "What are the Laws?" Two Concepts of Psychological Explanation', in F Keil \& RA Wilson (eds), Explanation and Cognition, Cambridge, MA: MIT Press.

Fetzer, J (2013), 'Carl Hempel', in EN Zalta (ed.), The Stanford Encyclopedia of Philosophy, from < http://plato.stanford.edu/archives/spr2013/entries/ hempel/>.

Glennan, S (1996), 'Mechanisms and the Nature of Causation', Erkenntnis, vol. 44, no. 1, pp. 49-71.

- (2002), 'Rethinking Mechanistic Explanation', Philosophy of Science, vol. 69, no. 3, pp. S342-S353.

Hempel, CG (1966), Philosophy of Natural Science, Englewood Cliffs: Prentice Hall.

Illari, P (2013), 'Mechanistic Explanation: Integrating the Ontic and Epistemic', Erkenntnis (Online First), from < http://link.springer.com/article/10.1007/ s10670-013-9511-y>.

Kim, J (1962), 'On the Logical Conditions of Deductive Explanation', Philosophy of Science, vol. 30, no. 1, pp. 286-91. 
McManus, F (2012), 'Development and Mechanistic Explanation', Studies in History and Philosophy of Biological and Biomedical Sciences, vol. 43, no. 1, pp. 532-41.

Nicholson, DJ (2012), 'The Concept of Mechanism in Biology', Studies in History and Philosophy of Biological and Biomedical Sciences, vol. 43, no. 1, pp. 152-63.

Ramsey, J (2008), 'Mechanisms and their Explanatory Challenges in Organic Chemistry', Philosophy of Science, vol. 75, pp. 970-82.

Rosch, E (1975), 'Cognitive Representations of Semantic Categories', Journal of Experimental Psychology: General, vol. 104, no. 3, pp. 192-33.

(1978), 'Principles of Categorization', in B Aarts, D Denison, E Keizer, \& G Popova (eds), (2004), Fuzzy Grammar: A Reader, Oxford University Press, pp. 91-108.

Soom, P (2012), 'Mechanisms, Determination and the Metaphysics of Neuroscience', Studies in History and Philosophy of Biological and Biomedical Sciences, vol. 43, pp. 655-64.

Sun T \& Walsh, CA (2006), 'Molecular Approaches to Brain Assymmetry and Handedness', Nature Reviews: Neuroscience, vol. 7, no. 8, pp. 655-62.

Stoyanov, Z, Decheva, L, Pashalieva, L, \& Nikolova, P (2012), 'Brain Asymmetry, Immunity, Handedness', Central European Journal of Medicine, vol. 7, no. 1, pp. $1-8$.

Woodward, J (2011), 'Scientific Explanation', in EN Zalta (ed.), The Stanford Encyclopedia of Philosophy, from <http://plato.stanford.edu/archives/ win2011/entries/scientific-explanation/>.

\section{Other Sources Consulted}

Craver, CF (2009), 'Mechanisms and Natural Kinds', Philosophical Psychology, vol. 22, no. 5, pp. 575-94.

Machamer, P, Darden, L, \& Craver, CF (2002), 'Thinking about Mechanisms', Philosophy of Science, vol. 67, no. 1, pp. 1-25.

Piccinini, G \& Craver, CF (2011), 'Integrating Psychology and Neuroscience: Functional Analyses as Mechanism Sketches', Synthese, vol. 183, no. 1, pp. 283-311. 
The ANU Undergraduate Research Journal

Raerinne, J (2011), 'Causal and Mechanistic Explanations', Acta Biotheor, vol. 59, no. 1, pp. 251-71.

Strevens, M (2012), 'Précis of Depth', Philosophy and Phenomenological Research, vol. 84, no. 2, pp. 447-60.

Waskan, J (2011), 'Mechanistic Explanation at the Limit', Synthese, vol. 183, pp. 389-408. 
This text taken from The ANU Undergraduate Research Journal Volume Five 2013, published 2014 by ANU Press, The Australian National University, Canberra, Australia. 